\title{
Correctness of Multiplicative (and Exponential) Proof Structures is $N L$-Complete
}

\author{
(rapport interne LIPN - Mars 2007)
}

\author{
Paulin Jacobé de Naurois` ${ }^{\star}$ and Virgile Mogbil ${ }^{\star}$ \\ LIPN - UMR7030, CNRS - Université Paris 13, \\ 99 av. J-B Clément, F-93430 Villetaneuse, France \\ denaurois@lipn.univ-paris13.fr \\ virgile.mogbil@lipn.univ-paris13.fr
}

\begin{abstract}
We provide a new correctness criterion for unit-free MLL proof structures and MELL proof structures with units. We prove that deciding the correctness of a MLL and of a MELL proof structure is $N L$-complete. We also prove that deciding the correctness of an intuitionistic multiplicative essential net is $N L$-complete.
\end{abstract}

\section{Introduction}

The proof nets [Gir87,DR89] of Linear logic (LL) are a parallel syntax for logical proofs without all the bureaucracy of sequent calculus. They are a non-sequential graph-theoretic representation of proofs, where the order in which some rules are used in a sequent calculus derivation, when irrelevant, is neglected. The unitfree multiplicative proof nets are inductively defined from sequent calculus rules of unit-free Multiplicative Linear Logic (MLL). A proof structure is freely built on the same syntax as proof nets, without any reference to a sequent calculus derivation.

In LL we are mainly interested in the following decision problems: Deciding the provability of a given formula, which gives the expressiveness of the logic; deciding if two given proofs reduce to the same normal form, i.e. the cut-elimination problem which corresponds to program equivalence using the Curry-Howard isomorphism; and deciding the correctness of a given proof structure, i.e. whether it comes from a sequent calculus derivation. For this last decision problem, one uses a correctness criterion to distinguish proof nets among proof structures. We recall the following main results [Kan92,Mai] and we complete (in bold) the correctness cases:

\begin{tabular}{|c|c|c|c|c|}
\hline \multicolumn{2}{|c|}{ fragment } & \multicolumn{3}{|c|}{ decision problem } \\
\hline & units & provability & cut-elimination & correctness \\
\hline \hline MLL & no & $N P$-complete & $P$-complete & \multirow{2}{*}{ NL-complete } \\
\hline MELL & yes & open & (at most non-elementary) & \\
\hline
\end{tabular}

* Work supported by project NO-CoST (ANR) 
Correctness is equivalent to provability for unit only MLL because proof nets are formulae syntactic trees. However it is not so obvious for the propositional case as one can observe following the long story of correctness criteria:

- Long-trip [Gir87] is based on travels and was the first one.

- Acyclic-Connected [DR89] is a condition is based on switchings i.e. the choice of one premise for each 8 connective. The condition is that all the associated graphs are trees. A naive implementation of Acyclic-Connected uses exponential time.

- Contractibility [Dan90] is done in quadratic time by repeating two graph rewriting rules until one obtains a simple node.

- Graph Parsing [Laf95] is a strategy for Contractibility which is implemented in linear time as a sort of unification [Gue99].

- Dominator Tree [MO00,MO06] is a linear time correctness criterion for essential nets, to which proof structures correctness reduces in linear time.

- Ribbon [Mel04] is a topological condition requiring homeomorphism to the disk.

For other fragments of Linear Logic, some of these criteria apply or are extended as for MELL ${ }^{1}$ [Dan90,GM01].

A feature of these criteria is that they successively lower the complexity of sequential, deterministic algorithms deciding correctness for MLL. Switching from proof structures to paired graphs, that is undirected graphs with a distinguished set of edges, we give a new correctness criterion for MLL and more generally for MELL. This new correctness criterion gives us a lower bound for the correctness decision problem for both MLL and MELL. This lower bound yields an exact characterization of the complexity of this problem, and induces naturally efficient parallel and randomized algorithms for it.

The paper is organized as follows: we recall preliminary definitions and results in linear logic and complexity theory in Section 1. Section 2 is devoted to the exposition of a new correctness criterion for unit-free MLL and MELL with units. We prove its $N L$-completeness in Section 3, and the $N L$-completeness of the criterion for IMLL in Section 4.

\section{Background}

\subsection{MLL and Proof Structures}

Roman capitals $A, B$ stand for MLL formulae, which are given by the following grammar, where $\otimes$ and $\ngtr$ are duals for the negation ${ }^{\perp}$, accordingly to De Morgan laws:

$$
F::=A\left|A^{\perp}\right| F \otimes F \mid F \otimes F
$$

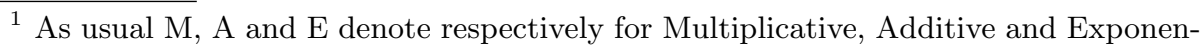
tial fragments of LL
} 
Greek capitals $\Gamma, \Delta$ stand for sequents, which are multiset of formulae, so that exchange is implicit. The MLL sequent calculus is given by the following rules:

$$
\overline{\vdash A, A^{\perp}}(a x) \quad \frac{\vdash \Gamma, C \vdash \Delta, C^{\perp}}{\vdash \Gamma, \Delta}(c u t) \quad \frac{\vdash \Gamma, A \vdash \Delta, B}{\vdash \Gamma, \Delta, A \otimes B} \otimes \frac{\vdash \Gamma, A, B}{\vdash \Gamma, A 8 B} \ngtr
$$

Definition 1. A MLL proof structure is a finite directed acyclic graph $(D A G)^{2}$ whose nodes, called links, are defined together with an arity and a coarity, i.e. a given number of incident edges called the premises of the node and a given number of emergent edges called the conclusions of the node. Moreover the proof structure edges are labelled by formulae and every edge is conclusion of exactly one link and premise of at most one link. The links of are the following:

\begin{tabular}{|l|c|c|c|c|c|c|c|c|c|}
\hline \multicolumn{2}{|c|}{ nodes } & \multicolumn{2}{c|}{$a x$} & \multicolumn{2}{c|}{ cut } & \multicolumn{2}{c|}{$\otimes$} & \multicolumn{2}{c|}{8} \\
\hline arity & edge labels & 0 & $\emptyset$ & 2 & $A, A^{\perp}$ & 2 & $A, B$ & 2 & $A, B$ \\
\hline coarity & edge labels & 2 & $A, A^{\perp}$ & 0 & $\emptyset$ & 1 & $A \otimes B$ & 1 & $A \ngtr B$ \\
\hline
\end{tabular}

We allow edges with a source but no target (i.e pending or dandling edges), they are called the conclusions of the proof-structure.

A MLL proof net is a MLL proof structure inductively defined as follows:

- an ax-link is a proof net with conclusions $A, A^{\perp}$,

- if $P$ is a proof net with conclusions $\Gamma, A, B$ then $P$ extended with a 8 -link of premises $A$ and $B$ is a proof net with conclusions $\Gamma, A \ngtr B$.

- if $P_{1}$ and $P_{2}$ are disjoint proof nets with respective conclusions $\Gamma, A$ and $\Delta, B$ then $P_{1} \cup P_{2}$ extended with $a \otimes$-link of premises $A$ and $B$ is a proof net with conclusions $\Gamma, A \otimes B, \Delta$.

It follows from the definition that MLL proof structures and proof nets have a non-empty set of conclusions, which corresponds to a MLL sequent. The inductive definition of MLL proof nets corresponds to a graph theoretic abstraction of the derivation rules of MLL; any proof net is sequentializable, i.e. corresponds to a MLL derivation: given a proof net $P$ of conclusion $\Gamma$, there exists a sequent calculus proof of $\vdash \Gamma$ which infers $P$.

Definition 2. A paired graph is an undirected graph $G=(V, E)$ with a set of pairs $C(G) \subseteq E \times E$ which are pairwise disjoint couples of edges with the same target, called a pair-node, and two (possibly distinct) sources called the premise-nodes.

$A$ switching $S$ of $G$ is the choice of an edge for every pair of $C(G)$. With each switching $S$ is associated a subgraph $S(G)$ of $G$ : for every pair of $C(G)$, erase the edges which are not selected by $S$. When $S$ selects the (abusively speaking) left edge of each pair, $S(G)$ is denoted as $G[\forall \mapsto \cdot \cdot]$. Also, $G[\forall \mapsto \because]$ stands for $G \backslash\left\{e, e^{\prime} \mid\left(e, e^{\prime}\right) \in C(G)\right\}$.

\footnotetext{
${ }^{2}$ For convenience the edges are oriented up-down, so we do not mention the orientation
} 
Let $R=(V, E)$ be a MLL proof structure. To $R$, we naturally associate the paired graph $G_{R}=\left(V, E^{\prime}\right)$ where $E^{\prime}$ is the set of non-pending edges of $E$ and $C\left(G_{R}\right)$ contains the premises of each \&-link of $R$ (Figure 1). For a pair of edges $(v, x),(w, x)$, we adopt the representation of Figure 1, where the two edges of the pair are joined by an arc.

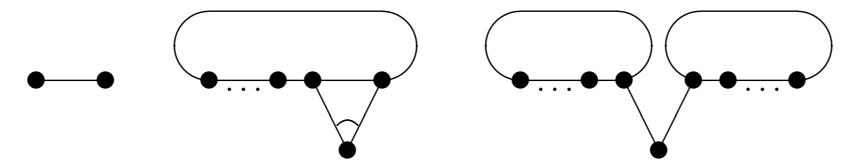

Fig. 1. Paired graph constructors associated to MLL proof nets: $a x$-link, $>$-link and $\otimes$-link.

We define the following graph rewriting rules $\rightarrow_{c}$ of Figure 2 on paired graphs where all the nodes are distinct and rule $\rightarrow_{R_{2}}$ applies only for a non-pair edge:

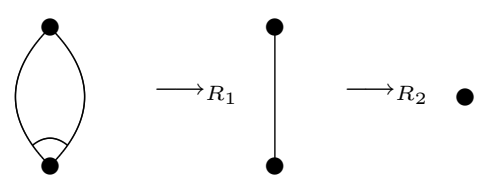

Fig. 2. Contraction rules $\rightarrow_{c}$

We denote by $G \rightarrow_{c} \bullet$ the fact that $G$ contracts to a single vertex with no edge.

Definition 3. A MLL proof structure $R$ is DR-correct if for all switching $S$ of $G_{R}$, the graph $S\left(G_{R}\right)$ is acyclic and connected.

$A$ MLL proof structure $R$ is contractile if $G_{R} \rightarrow_{c}^{*} \bullet$.

Theorem 1. [DR89,Dan90] A MLL proof structure $R$ is a MLL proof net iff $R$ is DR-correct iff $R$ is contractile ${ }^{3}$.

We define the following decision problem MLL-CORR:

Given: A MLL proof structure $R$

PRoBlem: Is $R$ a MLL proof net?

\subsection{MELL and Proof Structures}

The definition of MELL formulae follows that of MLL formulae in Section 1.1, with ! and ? duals for the negation ${ }^{\perp}$, as well as the neutral elements 1 and $\perp$ :

MELL:

$$
F::=A\left|A^{\perp}\right| F \otimes F|F \ngtr F| ! F|? F| 1 \mid \perp
$$

The MELL sequent calculus contains the rules of the MLL sequent calculus, as well as the following rules:

$\frac{\vdash \Gamma}{\vdash \Gamma, \perp} \perp \quad \frac{\vdash 1}{\vdash 1} \quad \frac{\vdash \Gamma}{\vdash \Gamma, ? A} ? W \quad \frac{\vdash \Gamma, ? A, ? A}{\vdash \Gamma, ? A} ? C \quad \frac{\vdash \Gamma, A}{\vdash \Gamma, ? A} ? D \quad \frac{\vdash ? \Gamma, A}{\vdash ? \Gamma, ! A} ! P$

${ }^{3}$ The criteria in [DR89,Dan90] are expressed for switchings and contraction rules for proof structures only. The equivalence with Definition 3 is left to the reader. 
Definition 4. MELL structures are defined similarly to MLL proof structures (Definition 1), with the additional links, where the ?W-link subsumes both ?W and $\perp$ rules:

\begin{tabular}{|c|c|c|c|c|c|c|c|c|c|c|c|}
\hline \multicolumn{2}{|c|}{ nodes } & \multicolumn{2}{|c|}{1} & \multicolumn{2}{|r|}{$? W$} & \multicolumn{2}{|r|}{$? C$} & \multicolumn{2}{|c|}{$? D$} & \multicolumn{2}{|c|}{$! P$} \\
\hline arity & edge labels & 0 & $\emptyset$ & & $\emptyset$ & 2 & $? A, ? A$ & 1 & $A$ & 1 & $A$ \\
\hline coarity & edge labels & 1 & 1 & 1 & $\perp$ or $? A$ & 1 & $? A$ & 1 & $? A$ & 1 & $! A$ \\
\hline
\end{tabular}

Definition 5. An exponential box is a MELL structure whose conclusions are all ?-formulae but one, its principal door, which is conclusion of a !P-link. Similarly, a weakening box is a MELL structure with a distinguished conclusion, its principal door, which is conclusion of a ?W-link. A box is either an exponential or a weakening box.

Definition 6. A MELL proof structure $(R, B)$ is given by a MELL structure $R$ and a box mapping $B$, which associates to any link $l$ of $R$ a box $b_{l}$ or $R$. Moreover, boxes may nest but may not partially overlap, and a unique exponential (respectively weakening) box is associated to each !P-(resp. ?W-)link. By convention, when a link belongs to several boxes, the mapping returns the innermost box to which it belongs, otherwise it returns $R$.

It follows from the definition that, for any $! P$ (respectively ?W)-link, the box mapping associates the exponential (resp. weakening) box to which it naturally corresponds. The whole proof structure $R$ is treated as a particular box, and is associated to all links that do not belong to any exponential or weakening box. Let $(R, B)$ be a MELL proof structure, with boxes $b_{1}, \ldots, b_{n}$. Let $b_{0}=R$. We define as follows the family $\mathcal{G}_{(R, B)}=\left\{G_{(R, B)}^{i}\right\}_{i=0 \ldots n}$ of paired-graphs:

- $G_{(R, B)}^{i}$ contains a node $l$ for every link $l$ of $R \backslash\{? W$-links $\}$ with $B(l)=b_{i}$, and an edge $\left(l, l^{\prime}\right)$ for all links $l, l^{\prime}$ of $R \backslash\{? W$-links $\}$ with $B(l)=B\left(l^{\prime}\right)=b_{i}$. $C\left(G_{(R, B)}^{i}\right)$ contains the premises of each 8 -link and ?C-link $l$ of $R$ with $B(l)=b_{i}$.

- Assume $b_{j}$ is an outermost box included in $b_{i}$. A node $\overline{b_{j}} \in G_{(R, B)}^{i}$ is associated to $b_{j}$, and an edge $\left(\overline{b_{j}}, l\right) \in G_{(R, B)}^{i}$ for all link $l$ conclusion of a link in $b_{j}$ and such that $B(l)=b_{i}$.

Essentially, $G_{(R, B)}^{i}$ is the paired graph corresponding to the box $b_{i}$, where all inner boxes are considered contracted to a single node. Moreover, for the sake of connectivity, the ?W-link (if there is any) corresponding to $b_{i}$ is removed.

Definition 7. A MELL proof structure $(R, B)$ with boxes $b_{1}, \ldots, b_{n}$ is contractile if $\forall i \in\{0, \ldots, n\}, G_{(R, B)}^{i} \rightarrow_{c}^{*} \bullet$.

As for MLL, one may inductively define particular MELL proof structures, called MELL proof nets, which exactly correspond to derivations in MELL sequent calculus. Theorem 2 allows to distinguish MELL proof nets among proof structures:

Theorem 2. [GM01] A MELL proof structure $(R, B)$ is a MELL proof net iff $(R, B)$ is contractile ${ }^{4}$.

${ }^{4}$ The criterion in [GM01] uses contraction rules for MELL proof structures only. As for Theorem 1, the proof of the equivalence with Definition 7 is left to the reader. 
We define the following decision problem MELL-CORR:

GIVEn: A MELL proof structure $(R, B)$

Problem: Is $(R, B)$ a MELL proof net?

\subsection{Intuitionistic Multiplicative Linear Logic and Essential Nets}

The intuitionistic fragment of MLL (IMLL) is the $(\otimes, \multimap)$-fragment of Linear logic, where linear implication is no more defined by $A \multimap B=A^{\perp} \ngtr B$ but is a connective. The sequent calculus corresponds to the MLL sequent calculus but with two-sided sequents (using linear negation) and at most one (distinguished) formula on the right.

For this sub-logic, Lamarche has proposed a version of proof structures called essential nets. They are built on the links given in Figure 3 where, to each $8^{+}$ labelled node, one associates a negatively-labelled node (left premise) called the sink of $p$. They also have a distinguished link called the root.

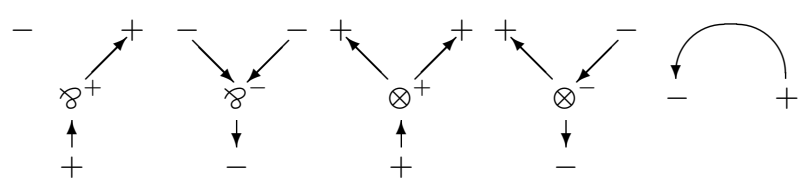

Fig. 3. Essential net links: $\succ^{+}, \succ^{-}, \otimes^{+}, \otimes^{-}$and Axiom

Definition 8. An essential net of a linearly balanced ${ }^{5}$ IMLL sequent is L-correct if it is acyclic, every node is reachable from the root, and every $8^{+}$-node $p$ satisfies the L-condition: every path from the root that reaches the sink of $p$ passes through $p$.

Lamarche has shown that the essential net of an IMLL sequent denotes a IMLL sequent derivation if and only if it is $L$-correct. For additional information on essential net correction, including translation to proof structures, we refer the reader to [MO06].

We define the following decision problem IMLL-CORR:

Given: A multiplicative essential net $N$ of a linearly balanced IMLL sequent Problem: Is $N$ correct?

\subsection{Complexity Classes and Related Problems}

We will mention several major complexity classes below $P$, some of which having natural complete problems that we will use in this paper. Let us briefly recall some basic definitions and results.

Definition 9. Complexity classes:

\footnotetext{
${ }^{5}$ I.e. every atom that occurs in the sequent does so exactly twice, once positively and once negatively
} 
- $A C^{0}$ is the class of problems solvable by a uniform family of circuits of constant depth and polynomial size, with NOT gates and AND, OR gates of unbounded fan-in.

- NC 1 is the class of problems solvable by a uniform family of circuits of logarithmic depth and polynomial size, with NOT, AND and OR gates of bounded fan-in.

- L is the class of problems solvable by a deterministic Turing machine which only uses a logarithmic working space.

- NL is the class of problems solvable by a non-deterministic Turing machine which only uses a logarithmic working space, such that:

1. If the answer is "yes," at least one computation path accepts.

2. If the answer is "no," all computation paths reject.

- coNL is the class of problems solvable by a non-deterministic Turing machine which only uses a logarithmic working space, such that:

1. If the answer is "yes," all computation paths accept.

2. If the answer is "no," at least one computation path rejects.

- $A C^{1}$ is the class of problems solvable by a uniform family of circuits of logarithmic depth and polynomial size, with NOT gates and AND, OR gates of unbounded fan-in.

Theorem 3. [Imm88,Sze87] $N L=c o N L$.

The following inclusion results are also well known:

$$
A C^{0} \subset N C^{1} \subset L \subset N L \subset A C^{1} \subset P,
$$

where it remains unknown whether any of these inclusions is strict.

It is important to note that Theorems 6 and 7 give $N L$-completeness results under constant-depth (actually $A C^{0}$ ) reductions. From (1) above, it should be clear to the reader that the reductions lie indeed in a class small enough for being relevant. For a good exposition of constant-depth reducibility, we refer the reader to [CSV84].

In the sequel, we will often use the notion of a path in a directed -or undirectedgraph. A path is a sequence of vertices such that there is an edge between any two consecutive vertices in the path. A path will be called elementary when any node occurs at most once in the path.

Let us now list some graph-theoretic problems that will be used in this paper.

Is TREe (IT)

Given: An undirected graph $G=(V, E)$

Problem: Determine whether $G$ is a tree

IT is $L$-complete under constant-depth reductions [JLM97].

Source-Target Connectivity (STCONN)

Given: A directed graph $G=(V, E)$ and two designated vertices $s$ and $t$

Problem: Is there a path from $s$ to $t$ in $G$ ?

STCONN is $N L$-complete under constant-depth reductions [JLL76]. 
Undirected Source-TARget Connectivity (USTCONN)

GIVEN: An undirected graph $G=(V, E)$ and two designated vertices $s$ and $t$ Problem: Do $s$ and $t$ belong to the same connected component of $G$ ?

USTCONN is $L$-complete under constant-depth reductions [Rei05].

Universal Source DAG (SDAG)

Given: A directed graph $G=(V, E)$ and a designated (source) vertex $s$

Problem: Is $G$ acyclic and is there a path from the source $s$ to each vertex ?

Proposition 1. SDAG $\in N L$.

Proof. Given $G=(V, E)$ a directed graph, its acyclicity can be expressed as follows:

$$
\forall(x, y) \in V^{2}: \neg \operatorname{STCONN}(G, x, y) \vee \neg \operatorname{STCONN}(G, y, x) .
$$

Since $N L=c o N L$ (Theorem 3) and STCONN $\in N L$, acyclicity is clearly in $N L$. Checking whether each vertex can be reached from the source vertex $s$ can also be done with STCONN subroutines, therefore SDAG is in $N L$.

Proposition 2. SDAG is coNL-hard under constant-depth reductions.

Proof. Let $\mathcal{L}$ be any language in $c o N L . \mathcal{L}$ is then decided by a non-deterministic Turing machine $\mathrm{M}$ in space less than $k \log (n)$ on inputs of size $n$, for some $k \geq 0$. Let $\mathcal{C}_{n}$ be the set of configurations of $\mathrm{M}$ of size less or equal to $k \log (n)$, and define $T=\left|\mathcal{C}_{n}\right|$. Clearly, $T \leq n^{k}$ is an upper bound for the computation time of $M$ on inputs of size $n$. Without loss of generality, we assume that every configuration of $M$ has at least one outgoing transition, possibly towards itself, and that the result of the computation is given by the state reached by $\mathrm{M}$ after exactly $T$ computation steps. A configuration is thus either accepting or rejecting.

Let us consider the following directed graph $G_{n}=\left(V_{n}, E_{n}\right)$, where:

$V_{n}=\bigcup_{c \in \mathcal{C}_{n}, t \in[0, T]}\{(c, t)\} \cup\left\{c_{A}\right\} \cup\left\{c_{R}\right\} \cup\{s\}$.

For $(c, t),\left(c^{\prime}, t+1\right) \in V_{n},\left(\left(c^{\prime}, t+1\right) \rightarrow(c, t)\right) \in E_{n}$ if and only if $c \rightarrow c^{\prime}$ is a transition of $\mathrm{M}$.

For $c \in \mathcal{C}_{n},\left(c_{A} \rightarrow(c, T)\right) \in E_{n}$ iff $c$ is an accepting configuration of M.

For $c \in \mathcal{C}_{n},\left(c_{R} \rightarrow(c, T)\right) \in E_{n}$ iff $c$ is a rejecting configuration of M.

$\left(s \rightarrow c_{A}\right) \in E_{n},\left(s \rightarrow c_{R}\right) \in E_{n}$.

A path $\left(c_{1}, t_{1}\right) \rightarrow \cdot \cdot \rightarrow\left(c_{k}, t_{k}\right)$ in $G_{n}$ follows by construction a sequence $t_{1}, \ldots, t_{k}$ that is strictly decreasing. Since there is no edge $(c, t) \rightarrow c_{A},(c, t) \rightarrow c_{R}$ nor $(c, t) \rightarrow s$, it is then clear that $G_{n}$ is acyclic.

Moreover, since every configuration of $M$ has at least one outgoing transition, every vertex $(c, t), t<T$ in $G_{n}$ has at least one parent node $\left(c^{\prime}, t+1\right)$. By induction on $t$, it follows that every vertex in $G_{n}$ is reachable from $s$. Therefore $G_{n}$ satisfies SDAG.

Let $x$ be an input of size $n$ to M. An initial configuration $c_{x} \in \mathcal{C}_{n}$ of $M$ is naturally associated to this input $x$. Consider now the directed graph $H_{n}^{x}=$ $G_{n} \cup\left\{\left(c_{x}, 0\right) \rightarrow c_{R}\right\}$. 
Then, $H_{n}^{x}$ satisfies SDAG if and only if $x \in \mathcal{L}$. Indeed, by Definition $9, x \in \mathcal{L}$ if and only if there exists no computation path $c_{x} \rightarrow \cdot \cdot \rightarrow c_{r}$ of length $T$ in $M$, where $c_{r}$ is a rejecting configuration. By construction of $G_{n}$, such a path corresponds to a path $\left(c_{r}, T\right) \rightarrow \cdot \rightarrow\left(c_{x}, 0\right)$ in $G_{n}$. Then $x \in \mathcal{L}$ if and only if there exists no path $c_{R} \rightarrow \cdots \rightarrow\left(c_{x}, 0\right)$ in $G_{n}$, if and only if $H_{n}^{x}$ is acyclic. Since $G_{n}$ satisfies SDAG, it follows that $H_{n}^{x}$ satisfies SDAG if and only if $x \in \mathcal{L}$.

Moreover, it is well known that the configuration graph of a Turing machine can be computed with constant-depth circuits. Computing $H_{n}^{x}$ from the configuration graph of $M$ requires only purely local rewriting rules, that can all be performed in parallel. Therefore, $H_{n}^{x}$ can also be computed with constant-depth circuits. $\square$ Propositions 1 and 2, and Theorem 3 yield the following result:

Theorem 4. SDAG is NL-complete under constant-depth reductions.

\section{New Correctness Criteria for MLL and MELL}

Definition 10. Let $G$ be a paired graph. The dependency graph $D(G)$ of $G$ is the directed graph $\left(V_{G}, E_{G}\right)$ defined as follows:

- $V_{G}=\{v \mid v$ is a pair-node in $G\} \cup\{s\}$.

- Let $x$ be a pair-node in $G$, with premise-nodes $x_{l}$ and $x_{r}$. The edge $(s \rightarrow x)$ is in $E_{G}$ if and only if:

1. There exists an elementary path $p_{x}=x_{l}, \ldots, x_{r}$ in $G[\forall \mapsto \nmid \cdot]$,

2. $x \notin p_{x}$, and

3. for all pair-node $y$ in $G, y \notin p_{x}$.

- Let $x$ be a pair-node in $G$, with premise-nodes $x_{l}$ and $x_{r}$, and let $y \neq x$ be another pair-node in $G$. The edge $(y \rightarrow x)$ is in $E_{G}$ if and only if:

1. There exists an elementary path $p_{x}=x_{l}, \ldots, x_{r}$ in $G[\forall \mapsto \cdot \cdot]$,

2. $x \notin p_{x}$, and

3. for every elementary path $p_{x}=x_{l}, \ldots, x_{r}$ in $G\left[\forall \mapsto \cdot{ }^{\circ}\right]$ with $x \notin p_{x}$, $y \in p_{x}$.

Lemma 1. Let $G$ and $H$ be paired graphs, with $G \rightarrow_{c} H$. Then, $G[\forall \mapsto \cdot \cdot] \rightarrow_{c}^{*}$ $H[\forall \mapsto \nmid \cdot]$, and $G[\forall \mapsto \nmid \cdot]$ is a tree if and only if $H[\forall \mapsto \nmid \cdot]$ is a tree.

Proof. If $G \rightarrow_{R_{1}} H$ denote by $v$ the redex pair-node in $G$, with premise $w$. The reduced pattern in $H$ is the non-pair edge $(v, w)$, therefore $G[\forall \mapsto \downarrow \cdot]=H[\forall \mapsto \nmid \cdot]$. If $G \rightarrow_{R_{2}} H$, it is clear that $G[\forall \mapsto \nmid \cdot] \rightarrow_{R_{2}} H[\forall \mapsto \nmid \cdot]$ with the same redex. It is also clear that rule $\rightarrow R_{2}$ preserves connectivity and acyclicity.

Lemma 2. If $G \rightarrow_{c}^{*} \bullet$ then $D(G)$ satisfies SDAG.

Proof. Since $\bullet[\forall \mapsto \mid \cdot]$ is a tree, by Lemma 1 so is $G[\forall \mapsto \nmid \cdot]$. Therefore, for any pair-node $x$ with premise-nodes $x_{l}$ and $x_{r}$ in $G$, there exists a unique elementary path $p_{x}=x_{l}-\cdots-x_{r}$ in $G[\forall \mapsto \nmid \cdot]$. It follows by construction of $D(G)$ that $x$ has at least one parent node in $D(G)$. Moreover, a path $x \rightarrow \cdot \cdot \rightarrow y$ in $D(G)$ induces by construction an elementary path $x_{l}-\cdots-y$ in $G[\forall \mapsto \cdot]$. Therefore a cycle $x \rightarrow \cdot \cdot \rightarrow y, y \rightarrow \cdot \cdot \rightarrow x$ in $D(G)$ induces a cycle $x_{l}-\cdots-y, y_{l}-\cdots-x$ in $G[\forall \mapsto l \cdot]$. Since $G[\forall \mapsto \nmid]$ is a tree, $D(G)$ is acyclic. Since every vertex of $D(G)$ but $s$ has at least one parent node and $D(G)$ is acyclic, $D(G)$ satisfies SDAG. 
Lemma 3. Let $G$ be a paired graph such that $G[\forall \mapsto \nmid \cdot]$ is a tree. If the dependency graph $D(G)$ of $G$ satisfies SDAG then $G \rightarrow_{c}^{*} \bullet$.

Proof. let $d(v)$, the depth of a pair-node $v \in G$, be the length of the longest path from the source $s$ of $D(G)$ to the vertex $v \in D(G)$. Assume that $D(G)$ satisfies SDAG, and let $X^{d}=\{x$ pair-node in $G \mid d(x)=d\}$ and $Y^{d}=\cup_{d^{\prime} \leqslant d} X^{d^{\prime}}$. By induction on the depth we prove that there exists a sequence of contractions $\mathcal{C}_{d}$ such that $G \rightarrow^{\mathcal{C}_{d}} G^{d}$ satisfies:

Each pair-node $y \in G$ s.t. $d(y) \leqslant d$ is contracted in $G^{d}$.

The proof by induction is as follows:

- For $d=1$, let $x \in X^{1}$, with premise-nodes $x_{l}$ and $x_{r}$. By definition of $X^{1}$, there exists an elementary path $p_{x}=x_{l}-\cdots-x_{r}$ in $G\left[\forall \mapsto: l^{\cdot}\right]$ such that $x \notin p_{x}$ and for any pair-node $y$ in $G[\forall \mapsto \nmid \cdot], y \notin p_{x}$. The same holds for the path $p_{x}=x_{l}-\cdots-x_{r}$ in $G$, with respect to any pair-node $y \in G$.

Let $\mathcal{E}_{x}^{1}=\left\{e\right.$ edge of $\left.p_{x} \mid x \in X^{1}\right\}$. The set of contractions $\mathcal{R}_{x}^{1}=\left\{e \rightarrow_{c} \bullet \mid e \in\right.$ $\left.\mathcal{E}_{x}^{1}\right\}$ contracts the edges of $p_{x}$, and let $\mathcal{R}^{1}=\cup_{x \in X^{1}} \mathcal{R}_{x}^{1}$. Clearly, $x_{l}=x_{r} \neq x$ in the contracted paired graph obtained from $G$ by $\mathcal{R}_{x}^{1}$. Since $x \notin p_{y}$ for any $y \in X^{1}$, the same holds for the contracted paired graph obtained from $G$ by $\mathcal{R}^{1}$.

Let $\mathcal{C}_{1}$ be the sequence $\mathcal{R}^{1}$, followed by the set of contraction rules of the pair-nodes $x \in X^{1}$. Define $G^{1}$ such that $G \rightarrow^{\mathcal{C}_{1}} G^{1}$. It is clear that $G^{1}$ satisfies (2).

- Assume by induction that there exists a sequence of contractions $\mathcal{C}_{d}$ such that $G \rightarrow^{\mathcal{C}_{d}} G^{d}$ satisfies (2).

Let $x \in X^{d+1}$, with premise-nodes $x_{l}$ and $x_{r}$.

Since $G \rightarrow^{\mathcal{C}_{d}} G^{d}$ and $G[\forall \mapsto \nmid \cdot]$ is a tree, Lemma 1 applies:

$$
G[\forall \mapsto \nmid \cdot] \rightarrow^{\mathcal{C}_{d}^{\prime}} G^{d}[\forall \mapsto \nmid \cdot], \text { and } G^{d}[\forall \mapsto \nmid \cdot] \text { is a tree. }
$$

By definition of $X^{d+1}$, there exists an elementary path $p_{x}=x_{l}-\cdots-x_{r}$ in $G[\forall \mapsto \nmid \cdot]$ such that $x \notin p_{x}$ and, for every pair-node $y \in G$ of depth $d(y)>d$, $y \notin p_{x}$.

Define $p_{x}^{d}$ such that $p_{x} \rightarrow \mathcal{C}_{d}^{\prime} p_{x}^{d}$. By (3), $p_{x}^{d}$ is an elementary path in $G^{d}[\forall \mapsto \nmid]$ such that $x \notin p_{x}^{d}$ and, for every pair-node $y \in G^{d}[\forall \mapsto \nmid \cdot]$ of depth $d(y)>d$, $y \notin p_{x}^{d}$. The same holds for $p_{x}^{d}$ in $G^{d}$, with respect to any pair-node $y \in G^{d}$, since, by induction, for any pair-node $y \in G^{d}, d(y)>d$.

Let $\mathcal{E}_{x}^{d+1}=\left\{e\right.$ edge of $\left.p_{x} \mid x \in X^{d+1}\right\}$. The set of contractions $\mathcal{R}_{x}^{d+1}=\left\{e \rightarrow_{c}\right.$ - $\left.\mid e \in \mathcal{E}_{x}^{d+1}\right\}$ contracts the edges of $p_{x}^{d}$, and let $\mathcal{R}^{d+1}=\cup_{x \in X^{d+1}} \mathcal{R}_{x}^{d+1}$. Clearly, $x_{l}=x_{r} \neq x$ in the contracted paired graph obtained from $G$ by $\mathcal{R}_{x}^{d+1}$. Since $x \notin p_{y}$ for any $y \in X^{d+1}$, the same holds for the contracted paired graph obtained from $G$ by $\mathcal{R}^{d+1}$.

Let $\mathcal{C}_{d+1}$ be the sequence $\mathcal{C}_{d}$, followed by $\mathcal{R}_{d+1}$, and followed by the set of contraction rules of the pair-nodes $x \in X^{d+1}$. Define $G^{d+1}$ such that $G \rightarrow^{\mathcal{C}_{d+1}} G^{d+1} . G^{d+1}$ satisfies (2). 
Since $D(G)$ satisfies SDAG, the maximal depth $m=\max \{d(x) \mid x \in D(G)\}$ is well-defined and every pair-node $x$ of $G$ belongs to $X^{m}$. Therefore, $G \rightarrow^{\mathcal{C}_{m}} G^{m}$ and $G^{m}$ satisfies (2). Since $G[\forall \mapsto \nmid \cdot]$ is a tree, by Lemma 1 so is $G^{m}[\forall \mapsto \nmid \cdot]=G^{m}$. It follows that $G \rightarrow_{c}^{*} \bullet$.

Lemmas 2 and 3 and Theorems 1 and 2 imply the Theorem:

Theorem 5 (Correctness Criteria).

$A$ MLL proof structure $R$ is a MLL proof net if and only if:

1. $D\left(G_{R}\right)$ satisfies SDAG, and

2. $G_{R}[\forall \mapsto \nmid \cdot]$ is a tree.

$A$ MELL proof structure $(R, B)$ with boxes $b_{1}, \ldots, b_{n}$ is a MELL proof net if and only if:

1. $\forall i \in\{0, \ldots, n\}, D\left(G_{(R, B)}^{i}\right)$ satisfies $\mathrm{SDAG}$, and

2. $\forall i \in\{0, \ldots, n\}, G_{(R, B)}^{i}[\forall \mapsto \nmid \cdot]$ is a tree.

\section{$3 \quad N L$-Completeness of the Criteria for MLL and MELL}

Let DePGRAPH be the function: $G \mapsto D(G)$, which associates its dependency graph to a paired graph $G$.

Lemma 4. DEPGRAPH $\in F L$.

Proof. The following functions can easily be computed in $F L$ :

$-G, x \in G \mapsto(G[\forall \mapsto \nmid \cdot]) \backslash\{x\}$

$-G, x \in G \mapsto(G[\forall \mapsto \because]) \backslash\{x\}$

$-G, x \in G, y \in G \mapsto(G[\forall \mapsto \nmid \cdot]) \backslash\{x, y\}$

Consider now the following algorithm for DePGRAPH:

INPUT $(G)$

FOR ALL $x$ pair-node in $G$, with premise-nodes $x_{l}$ and $x_{r}$ DO IF USTCONN $\left((G[\forall \mapsto \cdot \cdot]) \backslash\{x\}, x_{l}, x_{r}\right)$ THEN OUTPUT $(s \rightarrow x) \in D(G)$

FOR ALL ( $x$ pair-node in $G$, with premise-nodes $x_{l}$ and $x_{r}, y$ pair-node in $G$ ) DO IF $\neg \operatorname{USTCONN}\left((G[\forall \mapsto \nmid \cdot]) \backslash\{x, y\}, x_{l}, x_{r}\right)$

AND USTCONN $\left((G[\forall \mapsto \cdot \cdot]) \backslash\{x\}, x_{l}, x_{r}\right)$ THEN

OUTPUT $(y \rightarrow\{x\}) \in D(G)$.

- USTCONN $\left((G[\forall \mapsto \cdot:]) \backslash\{x\}, x_{l}, x_{r}\right)$ tests whether there exists an elementary path $p_{x}=x_{l}-\cdots-x_{r}$ such that $x \notin p_{x}$ and, for all pair-node $y$ in $G$, $y \notin p_{x}$.

$-\neg \operatorname{USTCONN}\left((G[\forall \mapsto \cdot \cdot]) \backslash\{x, y\}, x_{l}, x_{r}\right)$ tests whether any elementary path $p_{x}=x_{l}-\cdots-x_{r}$ such that $x \notin p_{x}$ contains $y$.

- USTCONN $\left((G[\forall \mapsto \cdot]) \backslash\{x\}, x_{l}, x_{r}\right)$ tests whether there exists a path $p_{x}=x_{l}-\cdots-x_{r}$ in $G^{\prime}$ such that $x \notin p_{x}$. From the previous point, if such a path $p_{x}$ exists, $y \in p_{x}$.

It follows that this algorithm computes DePGRAPH. Since USTCONN $\in L$, this algorithm belongs to $F L^{L}$ (the class of functions computable in logarithmic space, with oracles in $L$ ). Since $F L^{L}=F L$, DePGRAPH $\in F L$. 
Proposition 3. $M E L L-\mathrm{CORR} \in N L$.

Proof. Let $(R, B)$ be a MELL-proof structure with boxes $b_{1}, \ldots, b_{n}$. Each function $(R, B), i \in\{0, \ldots, n\} \mapsto G_{(R, B)}^{i}$ can be easily be computed in FL. Checking that $G_{(R, B)}^{i}[\forall \mapsto \cdot]$ is a tree is doable in $L$ since IT $\in L$. Checking that $D\left(G_{(R, B)}^{i}\right)$ satisfies SDAG can be done in $N L$, by composing the function DEPGRAPH in $F L$ (Lemma 4) with an $N L$ algorithm for SDAG (Theorem 4). Since the number of paired graphs $G_{(R, B)}^{i}$ is linearly bounded, it suffices to sequentially perform these tasks for $i=0, \ldots, n$, with a counter $i$ of logarithmic size.

Proposition 4. $M L L$-CORR is NL-hard under constant-depth reductions.

Proof. We actually reduce SDAG to MLL-CORR. Let $G$ be a directed graph with a distinguished vertex, and consider the proof structure $S_{G}$ defined as follows (see Figure 4), and let $G_{S_{G}}$ be its associated paired graph:

1. To any vertex $v$ of $G$, we associate a $\otimes$-link $\bar{v}$ with parent links $\bar{v}_{\text {in }}$ and $\bar{v}_{\text {out }}$.

2. If there are $i>0$ in-going edges to $v, \bar{v}_{i n}$ is a 8 -link of arity $i$, with parent links $\bar{v}_{i n}^{1}, \ldots, \bar{v}_{i n}^{i}$. If $v$ has no in-going edge, $\bar{v}_{i n}$ is one conclusion of an axiomlink $A x_{i n}^{v}$, the other conclusion of $A x_{i n}^{v}$ being a conclusion of $S_{n}^{x}$.

3 . If there are $j>0$ outgoing edges from $v, \bar{v}_{\text {out }}$ is a $\otimes$-link of arity $j$, with parent links $\bar{v}_{\text {out }}^{1}, \ldots, \bar{v}_{\text {out }}^{j}$. If $v$ has no outgoing edge, $\bar{v}_{\text {out }}$ is one conclusion of an axiom-link $A x_{\text {out }}^{v}$, the other conclusion of $A x_{\text {out }}^{v}$ being a conclusion of $S_{n}^{x}$.

4. To an edge $v \rightarrow w$ of $G$, we associate an axiom-link $A x^{(v \rightarrow w)}$ with conclusions $A x_{i n}^{(v \rightarrow w)}$ and $A x_{o u t}^{(v \rightarrow w)}$. Moreover, if $v \rightarrow w$ is the $k^{t h}$ outgoing edge from $v$, $A x_{i n}^{(v \rightarrow w)}$ is $\bar{v}_{\text {out }}^{k}$. If $v \rightarrow w$ is the $l^{t h}$ in-going edge to $w, A x_{\text {out }}^{(v \rightarrow w)}$ is $\bar{w}_{\text {in }}^{l}$.

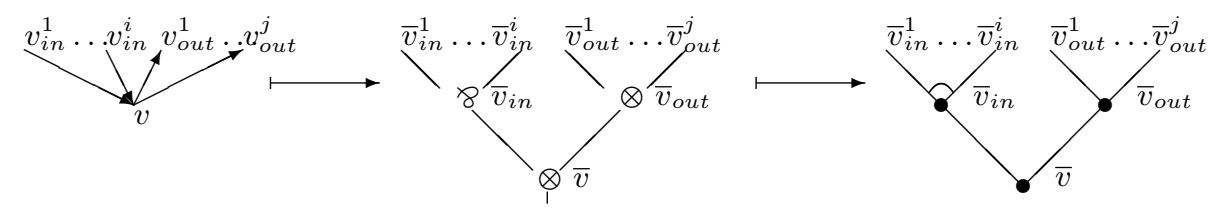

Fig. 4. Construction of $S_{G}$ and $G_{S_{G}}$

It is quite clear that this reduction can be computed by constant-depth circuits. We now claim that $S_{G}$ is correct if and only if $G$ satisfies SDAG.

Assume $G$ contains a cycle. There exists then an elementary path $p=x_{1} \rightarrow$ .. $\rightarrow x_{l}$, with $x_{l} \rightarrow x_{1} \in G$. Then, for any edge $x_{t} \rightarrow x_{t+1} \in p$, there exists a switching of the pair-node ${\overline{x_{t+1}}}_{i n}$ in $G_{S_{G}}$, which connects $\overline{x_{t}}$ and $\overline{x_{t+1}}$. Similarly for the edge $x_{l} \rightarrow x_{1} \in G$. Since $p$ is elementary, these pair-nodes are all different; therefore there exists cyclic switching of $G_{S_{G}}$ and $S_{G}$ is not correct.

Assume that $G$ is acyclic and has no unique source node. Then, there exist at least two nodes, $r$ and $s$, of arity 0 . Let $S^{\prime}$ be any switching of $G_{S_{G}}$, and assume 
that there exists an elementary path $p$ from $\bar{r}$ to $\bar{s}$ in $S^{\prime}$. Let $p^{\prime}=\bar{r}, \overline{x_{1}}, \ldots, \overline{x_{k}}, \bar{s}$ be the sequence of non pair-nodes of $p$ corresponding to vertices of $G$. $p^{\prime}$ follows by construction edges of $G$, accordingly to their orientation or not. Since $r$ and $s$ have arity 0 , there exist three nodes $\overline{x_{t}}, \overline{x_{t+1}}, \overline{x_{t+2}}$ in $p^{\prime}$ such that $\left(x_{t} \rightarrow x_{t+1}\right)$ and $\left(x_{t+2} \rightarrow x_{t+1}\right)$ are edges of $G$. By construction of $G_{S_{G}}, \overline{x_{t}}$ and $\overline{x_{t+2}}$ are then premise-nodes of the same pair-node $\bar{x}_{t+1}$ in in $G_{S_{G}}$, which contradicts that $p$ is a path in $S^{\prime}$. Therefore, $S^{\prime}$ is not connected, and $S_{G}$ is not correct.

Assume now that $G$ satisfies SDAG and let $d(v)$, the depth of a vertex $v$ of $G$, be the length of the longest path from the source $s$ of $G$ to $v$. Denote by $G^{d}$ the subgraph of $G$ consisting only in the vertices of depth less than $d$, and by $G_{S_{G}^{d}}$ the corresponding paired graph. It is easy to see that the rules of Figure 1 can be turned in a n-ary version, and that $G_{S_{G}^{d+1}}$ can be obtained from $G_{S_{G}^{d}}$ by these n-ary rules. By induction on $d$, it follows that $S_{G}$ is correct.

Since MLL is a subsystem of MELL, Propositions 3 and 4 immediately yield the following result:

Theorem 6. MLL-CORR and MELL-CORR are NL-complete under constantdepth reductions.

Remark 1. In the definition of the dependency graph, one may also choose to consider not only elementary paths, but any path. Surprisingly enough, the result holds also for this relaxed version of dependency, even though it is then less clear what dependency means.

\section{$4 \quad N L$-Completenesss of the Criterion for IMLL}

Proposition 5. IMLL - CORR $\in N L$.

Proof. For a given essential net $N$, denote by $r(N)$ its root. For a given $8^{+}$ link $x$ in $N$, denote by $s(x)$ its sink. Consider now the following algorithm for IMLL-CORR:

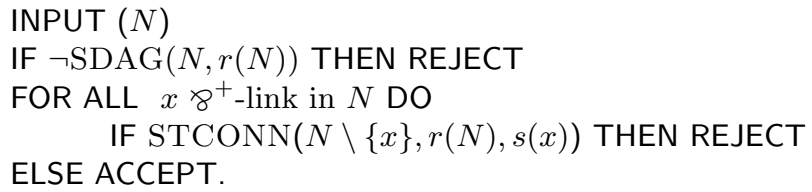

This algorithm checks that $N$ satisfies SDAG, and that the $L$-condition applies. Since $\mathrm{SDAG} \in N L$ and $\mathrm{STCONN} \in N L$, and $N L=c o N L$ (Theorem 3), this algorithm belongs clearly to $N L$.

Proposition 6. IMLL-CORR is NL-hard under constant-depth reductions.

Proof. We actually reduce SDAG to IMLL-CORR. Let $G$ be a directed graph with a distinguished vertex $s$, and consider the essential net $N_{G}$ defined as follows (see Figure 5): 
1. To any vertex $v$ of $G$ of arity $i>0$, we associate a $\otimes^{-}$-node $\bar{v}$, with parent node (right premise) $\bar{v}_{\text {in }}$ of polarity - and child node (left premise) $\bar{v}_{\text {out }}$ of polarity + . To $v$ of arity 0 we associate a node $\bar{v}=\bar{v}_{\text {out }}$ of polarity + .

2. If there are $i>0$ in-going edges to $v, \bar{v}_{i n}$ is a $8^{-}$-node of arity $i$, with parent nodes $\bar{v}_{i n}^{1}, \ldots, \bar{v}_{i n}^{i}$ of polarity - .

3. If there are $j>0$ outgoing edges from $v, \bar{v}_{\text {out }}$ is a $\otimes^{+}$-node of arity $j$, with child nodes $\bar{v}_{\text {out }}^{1}, \ldots, \bar{v}_{\text {out }}^{j}$ of polarity + .

4. If there is no outgoing edge from $v, \bar{v}_{\text {out }}$ is a $8^{+}$-node with child node (right premise) $\bar{v}_{\text {out }}^{1}$ of polarity + , and sink node (left premise) $\bar{v}_{\text {out }}^{\text {sink }}$, of polarity - . There is moreover an axiom-edge $\bar{v}_{\text {out }}^{1} \rightarrow \bar{v}_{\text {out }}^{\text {sink }}$.

5. Let $v \rightarrow w$ be an edge of $G$. Assume $v \rightarrow w$ is the $k^{\text {th }}$ outgoing edge from $v$, and the $l^{\text {th }}$ in-going edge to $w$. To $v \rightarrow w$, we associate an axiom-edge from $\bar{v}_{\text {out }}^{k}$ of polarity + to $\bar{w}_{\text {in }}^{l}$ of polarity - .

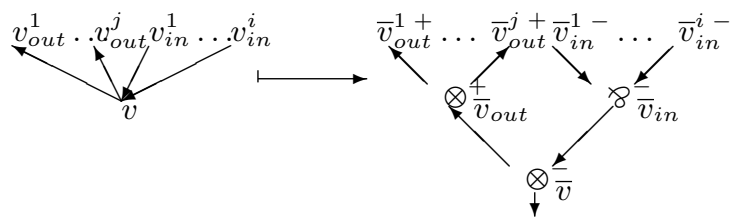

Fig. 5. Construction of $N_{G}$

Moreover, let $\bar{s}$ be the root of $N_{G}$. It is clear that the reduction is constantdepth. Since the only $8^{+}$-links of $N_{G}$ correspond to leaves of $G, N_{G}$ satisfies the $L$-condition by construction. Therefore, it is $L$-correct if and only if $G$ satisfies SDAG.

Propositions 5 and 6 immediately yield the following result:

Theorem 7. IMLL-CORR is NL-complete under constant-depth reductions.

\section{Conclusion}

Deciding the correctness of unit-free MLL proof structures, MELL proof structures, and unit-free IMLL essential nets where problems known to be decidable in deterministic, sequential linear time. We have shown their $N L$-completeness, thus establishing that it would be most unlikely to find better sequential deterministic algorithms. As a byproduct, we obtain efficient parallel algorithms for both problems, namely $A C^{1}$ algorithms. Moreover, since $N L=R L=Z P L$, we also obtain Monte-Carlo and Las-Vegas logarithmic space random algorithms for both problems. It remains to be checked whether our approach can be extended to MALL.

\section{Acknowledgments}

We are grateful to Harry Mairson for raising the question of the exact complexity of the correctness problems, and to the members of the No-Cost project for useful discussions and comments. 


\section{References}

[CSV84] Ashok K. Chandra, Larry J. Stockmeyer, and Uzi Vishkin. Constant depth reducibility. SIAM J. Comput., 13(2):423-439, 1984.

[Dan90] Vincent Danos. Une application de la logique linéaire à l'étude des processus de normalisation (principalement de $\lambda$-calcul). $\mathrm{PhD}$ thesis, Université Denis Diderot, Paris 7, 1990.

[DR89] Vincent Danos and Laurent Regnier. The structure of multiplicatives. Archive for Mathematical Logic, 28(3):181-203, 1989.

[Gir87] Jean-Yves Girard. Linear logic. Theoretical Computer Science, 50(1):1-102, 1987.

[GM01] Stefano Guerrini and Andrea Masini. Parsing MELL proof nets. Theoretical Computer Science, 254(1-2):317-335, 2001.

[Gue99] Stefano Guerrini. Correctness of multiplicative proof nets is linear. In Proc. of the annual Symp. on Logic in Computer Science (LICS'99), pages 454-463. IEEE Computer Society Press, 1999.

[Imm88] Neil Immerman. Nondeterministic space is closed under complementation. SIAM J. Comput., 17(5):935-938, 1988.

[JLL76] Neil D. Jones, Y. Edmund Lien, and William T. Laaser. New problems complete for nondeterministic logspace. Mathematical Syst. Theory, 10:1-17, 1976.

[JLM97] B. Jenner, K.-J. Lange, and P. McKenzie. Tree isomorphism and some other complete problems for deterministic logspace. DIRO 1059, Univ. de Montréal, 1997.

[Kan92] Max I. Kanovich. Horn programming in linear logic is np-complete. In Proc. of the annual Symp. on Logic in Computer Science (LICS'92), pages 200-210. IEEE Computer Society Press, 1992.

[Laf95] Yves Lafont. From proof-nets to interaction nets. In J.-Y. Girard, Y. Lafont, and L. Regnier, editors, Advances in Linear Logic, volume 222, pages 225-247. Cambridge University Press, 1995.

[Mai] Harry G. Mairson. Normalization bounds for multiplicative linear logic are axiom-sensitive. Presentation at GEOCAL'06 Workshop of Implicit Computational Complexity. Slides available at http://www-lipn.univparis13.fr/ baillot/GEOCAL06/SLIDES/Mairson.pdf.

[Mel04] Paul-André Melliès. A topological correctness criterion for non-commutative logic, volume 316 of London Mathematical Society Lecture Notes Series. Cambridge University Press, 2004.

[MO00] Andrzej Murawski and Luke Ong. Dominator trees and fast verification of proof nets. In Proc. of the annual Symp. on Logic in Computer Science (LICS'00), pages 181-191. IEEE Computer Society Press, 2000.

[MO06] Andrzej Murawski and Luke Ong. Fast verification of mll proof nets via imll. ACM Trans. Comput. Logic, 7(3):473-498, 2006.

[Rei05] Omer Reingold. Undirected st-connectivity in log-space. In Harold N. Gabow and Ronald Fagin, editors, STOC, pages 376-385. ACM, 2005.

[Sze87] Róbert Szelepcsényi. The method of forcing for nondeterministic automata. Bulletin of the EATCS, 33:96-99, 1987. 